\title{
IgA vasculitis with nephritis in children
}

\author{
Katarzyna Dyga ${ }^{1, B, D}$, Maria Szczepańska ${ }^{2, E, F}$ \\ ${ }^{1}$ Department of Pediatric Nephrology, Teaching Hospital No. 1, Medical University of Silesia, Zabrze, Poland \\ ${ }^{2}$ Chair and Department of Pediatrics, School of Medicine with the Division of Dentistry in Zabrze, Medical University of Silesia, Poland \\ A - research concept and design; $B$ - collection and/or assembly of data; $C$ - data analysis and interpretation; \\ $\mathrm{D}$ - writing the article; $\mathrm{E}$ - critical revision of the article; $\mathrm{F}$ - final approval of the article
}

\section{Address for correspondence \\ Katarzyna Dyga}

E-mail: katarzynadyga@wp.pl

\section{Funding sources}

None declared

\section{Conflict of interest}

None declared

Received on June 18, 2019

Reviewed on August 27, 2019

Accepted on September 24, 2019

Published online on April 30, 2020

\begin{abstract}
Immunoglobulin A vasculitis (IgAV), formerly known as Henoch-Schönlein purpura (HSP), is the most common form of systemic vasculitis in children. Although the first case of IgAV was described more than 200 years ago, its etiology still remains unclear. Nephrological symptoms are observed in $30-50 \%$ of children during the course of the disease, and in up to $91 \%$ of cases within 6 weeks of the onset of the first symptoms. Whereas other organ manifestations of IgAV are mostly benign and self-limiting, nephritis may lead to chronic kidney disease (CKD) and end-stage renal disease (ESRD). Appropriate treatment commenced early enough can stop the disease progression. However, even in severe cases there are no evidence-based guidelines, which makes the therapeutic decisions more difficult. In this article, which is the most up-to-date overview regarding IgAV, we discuss the disease's pathogenesis, the clinical forms of renal involvement in the course of the disease, the risk factors for adverse prognosis and treatment options in accordance with current recommendations.
\end{abstract}

Key words: children, nephritis, immunoglobulin A vasculitis

Cite as

Dyga K, Szczepańska M. IgA vasculitis with nephritis in children. Adv Clin Exp Med. 2020;29(4):513-519. doi: $10.17219 /$ acem/112566

DOI

10.17219/acem/112566

\section{Copyright}

Copyright by Author(s)

This is an article distributed under the terms of the

Creative Commons Attribution 3.0 Unported (CC BY 3.0)

(https://creativecommons.org/licenses/by/3.0/) 


\section{Introduction}

Immunoglobulin A vasculitis (IgAV), formerly known as Henoch-Schönlein purpura (HSP), is a type of smallvessel vasculitis and is the most common form of systemic vasculitis in children. ${ }^{1}$ The eponym "Henoch-Schönlein purpura" comes from the names of the 2 researchers, Johann Schönlein and his student Eduard Henoch, who first made a detailed analysis of this disease. According to the new nomenclature established during the International Chapel Hill Consensus Conference in 2012 (CHCC2012), the proper name of the disease is immunoglobulin A vasculitis (IgAV). The consensus to replace the former name (HSP) with IgAV was based on the pathophysiological feature of this disease whereby abnormal immunoglobulin A (IgA) is deposited in blood vessel walls. ${ }^{2}$ The most typical symptom of IgAV is skin involvement - mainly presenting as a palpable purpura which is often associated with systemic manifestations, including gastrointestinal pain and bleeding, arthralgia and/or arthritis and glomerulonephritis. Although most symptoms are self-limiting, renal disease is the most likely to result in long-term morbidity.

\section{Epidemiology}

The annual incidence of IgAV in children and infants is estimated to be 3-26.7 per 100,000 , depending on the country studied; it is 2-33 times more common in children than in adults. ${ }^{3}$ The disease occurs in children aged 3-15 years, though $90 \%$ of cases concern children under the age of 10 years; the mean age is 6 years. ${ }^{4,5}$ Immunoglobulin A vasculitis is more often diagnosed in boys, ${ }^{1,4-6}$ with a lower incidence in African-origin children compared with Caucasian or Asian. ${ }^{1}$ Immunoglobulin A vasculitis is also more common in children with familial Mediterranean fever. ${ }^{7}$

\section{Diagnostic criteria}

Immunoglobulin A vasculitis diagnosis should be based on the new classification criteria developed jointly by the European League Against Rheumatism (EULAR), the Pediatric Rheumatology International Trials Organization (PRINTO) and the Pediatric Rheumatology European Society (PRES) in 2008 and published in 2010, which have a sensitivity of $100 \%$ and a specificity of $87 \%$. The criteria include a diagnosis of non-thrombocytopenic skin purpura with lower limb predominance as the main symptom and 1 of 4 additional criteria: abdominal pain, arthritis or joint pain, symptoms of nephropathy (proteinuria $>0.3 \mathrm{~g} / 24 \mathrm{~h}$ or a urine protein/creatinine ratio (UP/UC ratio) $>30 \mathrm{mg} / \mathrm{mmol}$ on a spot morning sample or hematuria of $>5$ erythrocytes/high-power field), and a typical picture in histopathological examination of the skin showing leukocytoclastic vasculitis with predominant IgA deposits or kidney biopsy presenting proliferative glomerulonephritis with predominant IgA deposits. In cases of purpura with an atypical distribution, the presence of IgA deposits in the biopsy specimen is required. ${ }^{8}$ Ultrasonography is non-specific for IgAV (mesenteric lymphadenopathy and ascites detection), but along with computed tomography imaging is useful in diagnosing abdominal complications, such as intussusception, kidney parenchymal disease, and epididymal and testicular inflammation. ${ }^{9}$

\section{Pathogenesis}

Although the first case of this disease was described more than 200 years ago, its etiology still remains unclear. The current etiopathogenetic hypothesis for IgAV assumes an abnormal immune response to various antigens in genetically susceptible individuals. ${ }^{4}$ This theory is supported by the fact that IgAV mainly appears outside of the summer months, which is likely due to the relationship with infections and the lower risk of transmitting microbes among children during the summertime. ${ }^{1,4,10}$ In addition, symptoms of upper respiratory tract infection during the 3 weeks preceding the disease are reported in $90 \%$ of patients. ${ }^{4,11}$ In studies conducted independently by Calvino et al. and Saulsbury, a positive throat culture for group A beta-hemolytic streptococci (GAS) was found in 20-35\% of IgAV patients. ${ }^{5,12}$ Interestingly, Masuda et al. suggest that glomerular deposition of nephritis-associated plasmin receptor (NAPlr), a GAS antigen, may be responsible for some cases of IgAV nephritis. ${ }^{13}$ There is also an assumption that microorganisms could harbor antigenic structures resembling those of human small-vessel endothelial cells, consequently leading to the production of IgA cross-reactive anti-endothelial cell antibodies (AECA), which promote vascular damage by the activation of the complement system and antibody-dependent cellular cytotoxicity (ADCC). ${ }^{14}$

In the literature, non-infectious agents are also reported to initiate the development of IgAV, such as medications (e.g., non-steroidal anti-inflammatory drugs (NSAIDs)), angiotensin converting enzyme inhibitors (ACEi), angiotensin receptor blockers (ARB), vaccines (e.g., the influenza vaccine or the hepatitis $A$ and $B$ vaccines) and malignancies, in this case mainly affecting adults (e.g., non-small cell lung cancer or prostate cancer). ${ }^{15,16}$

It is also possible that there is a genetic predisposition to developing IgAV and subsequent renal involvement. The genetic studies carried out so far indicate that the HLA-DRB1*01:03 allele in Europeans and the HLA$B * 41: 02$ allele in Caucasians are associated with increased susceptibility to IgAV. ${ }^{17}$ López-Mejías et al. reported that a protective effect against the development of IgAV 
appears to exist in Caucasians with the HLA-DRB1:03 phenotype. ${ }^{18}$ Moreover, several molecular and genetic polymorphisms relating to disease susceptibility, severity and/or the risk of renal involvement have recently been described. An increased risk of nephritis in the course of IgAV is associated with the polymorphism of genes encoding vascular endothelial growth factor (VEGF), interleukin 1 receptor antagonist (IL-1Ra), interleukin $1 \beta$ (IL-1 $\beta$ ) and interleukin 8 (IL-8) and the presence of the HLA-B35 allele. ${ }^{19,20}$ However, most of the above studies were conducted on a relatively small number of patients, which means they lack the power to be definitive or necessarily applicable to all racial groups. ${ }^{21}$

Although the detailed pathogenic mechanism of IgAV with nephritis (IgAVN) has not been fully elucidated, it seems to be identical to IgA nephropathy (IgAN) with only quantitative differences, possibly accounting for the different clinical presentation with more acute signs of nephritis in IgAV and a more insidious onset of IgAN. However, the latest hypothesis, created by Heineke et al., suggests that renal and systemic symptoms in IgAV, IgAVN and IgAN may have different origins. ${ }^{22}$ Moreover, the authors anticipated that IgAVN is a dual disease which has components of both IgAV and IgAN.

The current hypothesis for glomerulonephritis in IgAN and IgAVN assumes that the symptoms are due to the deposition of immune complexes in small blood vessels (predominantly capillaries, venules or arterioles) which mainly consist of subclass 1 of immunoglobulin A, IgA1. The proposed mechanism of this phenomenon includes the disturbance of the glycosylation of oligosaccharides located in the hinge region of IgA 1 by the failure of galactose to attach to the $\mathrm{N}$-acetylgalactosamine residues found at this site. ${ }^{23}$ The primary defect that leads to the production of such abnormally glycosylated IgA1 is probably heritable $\mathrm{e}^{24,25}$ and might be due to a reduced activity of core 1 $\beta 1,3$-galactosyltransferase (C1GalT1) and an elevated expression and activity of $\alpha-\mathrm{N}$-acetylgalactosaminide $\alpha-2,6$ sialyltransferase 2 (ST6GalNAc-II) in peripheral B cells. ${ }^{23,26}$ There is also a hypothesis that mucosal infection and and concomitant interleukin 6 (Il-6) and interleukin 4 (Il-4) production may lead to aberrant glycosylation by modifying the glycosylation machinery. ${ }^{26}$

These aberrantly glycosylated IgA1 antibodies, called galactose-deficient IgA1 (Gd-IgA1), have been shown to form immune complexes with naturally occurring anti-glycan IgA1 or IgG antibodies, whose origin is poorly understood. ${ }^{22,23}$ It was noted that all IgAV patients have IgA1-containing circulating immune complexes (CIC) of low molecular mass, but only those with nephritis have additional high-molecular-mass IgA1- or IgG-containing CICs. ${ }^{16,23}$ There is an assumption that the presence of IgG in immune complexes facilitates deposition on the glomerular mesangium. ${ }^{22}$ It was also observed that IgA1containing immune complexes are present at higher concentrations in the urine of patients with IgAN and IgAVN and can form a specific marker for disease activity and/or severity in these patients. ${ }^{23,27}$

Due to the large size of CICs, their binding to the asialoglycoprotein receptors on hepatocytes (ASGP-R) and their internalization and degradation by hepatic cells are inhibited, which leads to their increased amounts in circulation. ${ }^{16}$ In the kidneys they bind to transferrin receptor (CD71, TfR) on mesangial cells, ${ }^{28,29}$ which enhances immune complex deposition and stimulates mesangial cells to proliferate and overproduce cellular matrix components and to secrete chemokines such as transforming growth factor beta (TGF $\beta$ ) or tumor necrosis factor alpha $\left(\right.$ TNF- $\alpha$ ) - which alter podocyte function ${ }^{30}$ - and IL-8, which recruits and activates polymorphonuclear neutrophils (PMNs). ${ }^{31}$ Accompanying glomerular complement activation through both the alternative and mannan-binding lectin pathways ${ }^{32}$ initiates the inflammatory cascade and enhances glomerular injury, contributing to the development of proliferative glomerulonephritis. Additionally, recent reports have assumed that IgA immune complexes directly induce neutrophils' migration and activation into affected tissues by binding to their IgA receptor, Fc $\alpha$ RI, worsening tissue damage. ${ }^{22}$

\section{Nephropathy}

The course of IgAV is usually mild and self-limiting, but in $30-50 \%$ of patients it may lead to renal complications, ${ }^{5,6,12}$ which vary from asymptomatic microscopic hematuria with or without proteinuria and macroscopic hematuria, through fully expressed nephritic or nephrotic syndrome to rapidly progressive glomerulonephritis (RPGN). As for hypertension, it may be isolated or related to renal impartment. ${ }^{7}$ The most often symptom of nephritis is isolated microscopic hematuria with or without proteinuria. ${ }^{5,12}$ According to Pohl, either nephritic or a nephrotic syndrome develop in about $20 \%$ of IgAVN patients (7\% of all IgAV cases). ${ }^{6}$

Nephrological symptoms usually appear early: in the $1^{\text {st }}$ month of the disease in $85 \%$ of cases, within 6 weeks in $91 \%$ and within 6 months of the onset of the first symptoms in $97 \%$ of cases. ${ }^{33}$ It is noteworthy that, according to several retrospective studies, nephritis never precedes the onset of skin lesions. ${ }^{5,12}$ Moreover, Saulsbury reports that the incidence of nephritis is significantly lower in children under the age of 2 years than in older children. ${ }^{5}$

The risk factors for developing nephropathy in the course of IgAV, according to a meta-analysis carried out by Chan et al., include male gender, an age of over 10 years, the presence of severe gastrointestinal symptoms (abdominal pain, gastrointestinal bleeding and ischemic intestinal injury), persistent purpura, the occurrence of relapses, arthritis/arthralgia, and certain laboratory abnormalities, such as leukocytosis above $15 \times 10^{9} / \mathrm{L}$, thrombocytosis above $500 \times 10^{9} / \mathrm{L}$, elevated serum antistreptolysin $\mathrm{O}$ 
(ASO) titer, and decreased serum c3 of the complement concentration. $^{34}$

A kidney biopsy in IgAV should be considered when acute renal impairment or nephritic syndrome is observed at the initial stage; moreover, when nephrotic syndrome with normal renal function persists for 4 weeks, nephrotic range proteinuria (a UP/UC ratio $>250 \mathrm{mg} / \mathrm{mmol}$ ) is observed for 4-6 weeks or persistent proteinuria (a UP/UC ratio $>100 \mathrm{mg} / \mathrm{mmol}$ ) occurs for more than 3 months. ${ }^{7}$ Interestingly, a study carried out by Halling et al. revealed that severe morphological changes found in renal biopsy occurred not only in patients with proteinuria in the nephrotic range but also in those with mild proteinuria. This observation allowed the authors to conclude that the clinical findings at the onset of the disease cannot predict the morphological changes. ${ }^{35}$ Likewise, a long-term study conducted by Ronkainen et al. confirmed that severity of findings on the first kidney biopsy did not correlate with the risk of a poor outcome (hypertension, persistent proteinuria or end-stage renal disease (ESRD)). ${ }^{36}$

As an alternative to kidney biopsy, Hara et al. showed that the podocyturia as well as hematuria and proteinuria had significant correlations with acute renal lesions - such as acute extracapillary, intracapillary and tubulointerstitial lesions - in biopsy. They also suggested that the cumulative excretion of urinary podocytes may be a predictor for histologic disease progression among children with IgAN and IgAVN. ${ }^{37}$

In a review conducted by Jelusic et al., the authors emphasized the role of appropriate histological classification in establishing guidelines for treating patients with IgAVN. However, a current problem of classifying IgAVN is that the classifications have not been validated in children or they have only been validated in IgAN. ${ }^{38}$ It is worth pointing out that, compared to IgAN, in IgAVN renal biopsy samples show increased endocapillary proliferation, epithelial crescents, neutrophil infiltration, perivascular glomerular IgA, subendothelial/subepithelial IgA deposits and fibrin deposits, ${ }^{6,16,38,39}$ which indicates the use of a different classification.

Currently, histological changes are graded in a classification which was published in 1977 by the International Study for Kidney Disease in Children (ISKDC), with 6 categories (I-V, according to the presence and number of crescents, and VI, for a membranoproliferative aspect). In this classification, only mesangial proliferation and the percentage of crescents is used to estimate disease severity and to predict outcome. However, the latest retrospective study, carried out on adults with IgAVN by Huang et al., showed that patients with more crescents had more severe renal manifestations and worse treatment response, whereas the proportion of crescents did not correlate with a higher risk for ESRD or a $50 \%$ decline in renal function. ${ }^{40}$ According to Davin, the main failing of the ISKDC classification is that it does not consider some other important prognostic factors, such as mesangial and endocapillary hypercellularity, tubular atrophy, interstitial fibrosis, interstitial inflammation, crescent features (fibrotic or not), segmental and global glomerulosclerosis and arterio- and arteriolosclerosis. ${ }^{41}$

Due to these all disadvantages of the ISKDC, some investigators prefer to apply the Oxford classification in updated in 2016 form called the MEST-C score, which includes 5 parameters: mesangial hypercellularity (M), endocapillary hypercellularity (E), segmental glomerulosclerosis $(\mathrm{S})$, tubular atrophy/interstitial fibrosis $(\mathrm{T})$, and cellular/fibrocellular crescents (C). However, this classification was originally created for IgAN and is currently not recommended for use in IgAVN. ${ }^{38,39}$ In 2017, $\mathrm{Xu}$ et al. estimated the value of the Oxford classification in children with IgAVN on the basis of a retrospective study including 104 patients. They found that segmental glomerulosclerosis (S) was strongly associated with impaired renal function and tubular atrophy/interistitial fibrosis (T) was significantly associated with proteinuria and clinical remission. The authors suggested that this classification may help to identify histological variables that have independent predictive value for a patient's response to therapy. ${ }^{42}$ Moreover, the latest study, conducted by Huang et al. on a larger group of IgAVN patients, revealed that the assessment of segmental glomerulosclerosis was an independent risk factor for renal endpoints, defined as a $\geq 30 \%$ reduction in baseline estimated glomerular filtration rate in 2 years or a doubling of serum creatinine or ESRD, and confirmed that an updated Oxford classification can be valuable in predicting renal outcomes in IgAVN. ${ }^{43}$ The classification developed by Koskela et al. in 2017 is the modified semiquantitative classification (SQC), which includes activity index, chronicity index and focal or diffuse mesangial proliferation. The authors demonstrated that the SQC was more coherent and more sensitive in terms of predicting IgAVN patient outcomes than the ISKDC. ${ }^{44}$ Although their research revealed promising results, a study on a larger number of patients is needed to properly validate this classification. ${ }^{38}$

\section{Treatment}

Treatment decisions in IgAV are difficult due to the large proportion of patients with a favorable prognosis and an unpredictable course in individual patients. Most reports in the literature consist of series or retrospective studies involving small numbers of patients, which makes the creation of standards of treatment more difficult.

In 2009, Zaffanello and Fanos published a review of literature from 1988 to 2008, which included reports of treatment with prednisolone, methylprednisolone, cyclophosphamide, azatioprine, cyclosporine, dypiridamole, warfarine and plasma exchange. The authors concluded that there was a lack of properly designed, randomized, placebo-controlled trials which could serve as a basis 
for appropriate standards of treatment for patients with IgAVN. ${ }^{45}$

In 2012, Kidney Disease: Improving Global Outcomes (KDIGO) published a practical guideline for the treatment of glomerulonephritis. According to their recommendations, in the case of persistent proteinuria of more than $0.5-1 \mathrm{~g} /$ day $/ 1.73 \mathrm{~m}^{2}$ in patients with IgAV, treatment with ACEi or ARB should be administered for 3-6 months, due to the renoprotective effects of these drugs, including delayed progression of renal failure and reduction of proteinuria. When this treatment is ineffective and the estimated glomerular filtration rate (eGFR) value remains higher than $50 \mathrm{~mL} / \mathrm{min} / 1.73 \mathrm{~m}^{2}$, it is recommended to use the procedure as in IgA nephropathy (IgAN), involving the introduction of steroid therapy for a period of 6 months. In children with glomerular crescents in renal biopsy (more than 50\% of glomeruli) and clinical manifestations of nephrotic syndrome and/or worsened renal function, the recommended treatment is similar of that for crescent IgA nephropathy, involving the use of steroids with simultaneous application of cyclophophamide (CYP). ${ }^{46}$ Kidney Disease: Improving Global Outcomes do not advise the use of steroids to prevent the development of nephropathy in the course of IgAV. Similarly, in several other studies it was demonstrated that steroid therapy does not protect against kidney involvement in the course of IgAV. ${ }^{5,45,47,48}$ However, these guidelines recommend an approach similar to treatment of IgAN patients, without taking into account the more acute onset of IgAV nephritis with more aggressive lesions in renal histology. In the opinion of Davin et al. the approach proposed by KDIGO may lead to undertreatment, mainly because acute and potentially aggressive glomerular inflammation either goes untreated or its immunosuppressive treatment is delayed for several months. ${ }^{49}$ Interestingly, the largest longitudinal observational study, including 506 patients with IgAN and 161 patients with IgAV conducted by The Cure Glomerulonephropathy Network (Cure GN), revealed that in comparison to patients with IgAN, those with IgAVN were more likely to receive immunosuppressive therapy (79.5\% vs 54\%; p < 0.001), of which $35 \%$ were being treated with $\geq 2$ immunosuppressive agents at the time of diagnosis. ${ }^{50}$ This fact may indirectly suggest that a more severe course of IgAV requires more aggressive treatment than IgAN. The latest nationwide, longitudinal follow-up study from Finland confirms that early treatment may have a favorable effect on final renal prognosis. ${ }^{51}$

Recently, the European initiative SHARE (Single Hub and Access point for paediatric Rheumatology in Europe) released the long-awaited international recommendations for the diagnosis and treatment of IgAV. The panel of experts divided IgAV with nephritis into 3 categories, depending on the severity: mild, moderate and severe. For patients with mild IgAVN (normal eGFR and mild proteinuria - a UP/UC ratio $<100 \mathrm{mg} / \mathrm{mmol}$ - or moderate proteinuria - a UP/UC ratio of 100-250 $\mathrm{mg} / \mathrm{mmol})$, SHARE recommends the use of oral prednisolone as a first-line treatment. However, the addition of other immunosuppressive agents, such as azathioprine (AZA), mycophenolate mofetil (MMF) or pulse iv. methylprednisolone (MP), may be considered as a second-line treatment. In moderate IgAVN $(<50 \%$ crescents on renal biopsy and impaired eGFR or severe persistent proteinuria - a UP/UC ratio $>250 \mathrm{mg} / \mathrm{mmol}$ for at least 4 weeks), oral prednisolone or pulse iv. methylprednisolone should be used as first-line treatment. In this case, the addition of AZA, MMF or iv. cyclophosphamide (CYP) may be used in first- or secondline treatment according to the histopathological findings of renal biopsy. In severe IgAVN $(>50 \%$ crescents on renal biopsy and impaired eGFR or severe persistent proteinuria) patients should be treated with iv. CYP and pulsed $\mathrm{MP}$ and/or oral prednisolone like patients with other forms of severe systemic small-vessel vasculitis. According to these recommendations patients with persistent proteinuria (a duration $>3$ months) should be treated with ACEi or ARB irrespective of whether they are receiving prednisolone or other immunosuppressive treatment. ${ }^{52}$

Both KDIGO and the latest SHARE recommendations are based mainly on expert opinions, because there is a lack of high-level controlled trials for severe IgAVN. Many authors emphasize the need to conduct well-designed, multicenter studies to provide evidence-based recommendations. ${ }^{39,45,46,52}$

\section{Prognosis}

The progression of renal impairment in the course of IgAV is unpredictable in patients at the individual level, and active renal disease may develop after an initially mild nephritis or many years after stable, minor urinary abnormalities. ${ }^{36,39,53,54}$ A study conducted by Nussinovitch et al. suggests that even patients with no urinary abnormalities at onset may develop hypertension later on. ${ }^{54}$ The occurrence of nephrological symptoms in some cases even long after the diagnosis of IgAV may be explained by the finding that the majority of children who underwent kidney re-biopsy within 2-9 years still have IgA deposits. ${ }^{21}$

While other organ manifestations of IgAV are mostly benign and self-limiting, nephritis might lead to chronic kidney disease (CKD) and ESRD. Long-term renal outcome correlates to the severity of the initial clinical presentation and to the extent of renal biopsy changes. ${ }^{35,39,53}$ A systemic review carried out by Narchi confirmed that the risk of long-term renal impairment, defined as persistent nephritic or nephrotic syndrome, renal insufficiency or hypertension, was low (1.6\%) in patients with only isolated proteinuria or hematuria, but was much higher (19.5\%) if the initial presentation was complicated by nephritic or nephrotic syndrome, with the risk being 2.5 times greater in women than in men. ${ }^{33}$ Other studies evaluating patients from tertiary care centers with pediatric 
nephrology wards found that the patients with nephritic, nephrotic or nephritic/nephrotic syndrome at onset had an even higher risk of long-term renal impairment, estimated at $35-44 \% .^{36,53}$

Adverse prognostic factors for ESRD in adults with IgAV include renal dysfunction which persists from the onset of the disease (a creatinine level $>1.35 \mathrm{mg} / \mathrm{dL}$ ), proteinuria of over $1 \mathrm{~g} /$ day, macroscopic hematuria, hypertension and some degree of interstitial fibrosis, the presence of sclerotic glomeruli and fibrinoid necrosis on histopathology from a kidney biopsy. ${ }^{55,56}$

Immunoglobulin A vasculitis recurs in about $1 / 3$ of all cases, ${ }^{5}$ generally within 4 months of the resolution of the original symptoms. Recurrences are more frequent in patients with renal involvement, but are usually milder and of shorter duration than the original episode..$^{5,12,21}$

The follow-up period for children presenting with IgAV should last at least 6-12 months and should include regular urine testing for proteinuria and hematuria as well as blood pressure monitoring. ${ }^{33,52}$ According to a review carried out by Narchi, there is no need to follow up after the first 6 months with patients whose urinalysis remains normal, but measurements of serum urea and creatinine need to be continued in the presence of persistent isolated hematuria and/or proteinuria. ${ }^{33}$

Women with a history of IgAV during childhood are at a higher risk of complications such as proteinuria and hypertension during pregnancy and should be monitored closely. ${ }^{33,36,53}$ In a study carried out by Ronkainen et al., up to $70 \%$ of pregnancies had complications of hypertension and/or proteinuria, even in the absence of active renal disease, which led to the conclusion that all women with a history of IgAV and even mild renal symptoms should be carefully observed, not only during pregnancy but also afterwards. $^{36}$

Regarding the risk of relapse of IgAV after kidney transplantation, depending on the source it is $33 \%$ to $50 \%,{ }^{15}$ whereas the risk of graft loss from the reccurence of the disease, as reported by Kanaan et al, is 7.5\% during 10 -year follow-up period. ${ }^{57}$

\section{Conclusions}

The authors emphasize that whereas other organ manifestations of IgAV are mostly benign and self-limiting, nephritis might lead to CKD, in its terminal stage as well. Appropriate treatment commenced early enough can stop the progression of the disease. However, even for the most severe cases, there are no evidence-based guidelines, which makes therapeutic decisions more difficult.

\section{ORCID iDs}

Katarzyna Dyga (D) https://orcid.org/0000-0002-7172-9179 Maria Szczepańska (D) https://orcid.org/0000-0002-6772-1983

\section{References}

1. Gardner-Medwin JM, Dolezalova P, Cummins C, Southwood TR. Incidence of Henoch-Schönlein purpura, Kawasaki disease and rare vasculitides in children of different ethnic origins. Lancet. 2002;360(9341): 1197-1202.

2. Jannette JC, Falk RJ, et al. 2012 Revised International Chapel Hill Consensus Conference of Nomenclature of Vasculitides. Arthritis Rheum. 2013;65(1):1-11.

3. Piram M, Mahr A. Epidemiology of immunoglobulin A vasculitis (Henoch-Schönlein): Current state of knowledge.CurrOpin Rheumatol. 2013;25(2):171-178.

4. Piram M, Maldini C, Biscardi S, et al. Incidence of IgA vasculitis in children estimated by four-source capture-recapture analysis: A population-based study. Rheumatology (Oxford). 2017;56(8):1358-1366.

5. Saulsbury FT. Henoch-Schönlein purpura in children. Report of 100 patients and review of the literature. Medicine (Baltimore). 1999;78(6): 395-409.

6. Pohl M. Henoch-Schönlein purpura nephritis. Pediatr Nephrol. 2015; 30(2):245-252.

7. McCarthy HJ, Tizard EJ. Clinical practice: Diagnosis and management of Henoch-Schönlein purpura. Eur J Pediatr. 2010;169(6):643-650.

8. Ozen S, Pistorio A, lusan SM, et al. EURAL/PRINTO/PRES criteria for Henoch-Schöenlein purpura, childhood polyarteritis nodosa, childhood Wegener granulomatosis and childhood Takayasu arteritis: Ankara 2008. Part II: Final classification criteria. Ann Rheum Dis. 2010; 69(5):798-806.

9. Singhal M, Gupta P, Sharma A. Imaging in small and medium vessel vasculitis. Int J Rheum Dis. 2019;22(Suppl 1):78-85.

10. Hwang HH, Lim IS, Choi BS, Yi DY. Analysis of seasonal tendencies in pediatric Henoch-Schönlein purpura and comparison with outbreak of infectious diseases. Medicine (Baltimore). 2018;97(36): e12217.

11. Jamrozik A, Sybilski A, Pohorecka M, Patena K. Zespół SchönleinaHenocha - nowe wyzwania diagnostyczne w starej chorobie. Pediatr Med Rodz. 2012;8(3):214-221.

12. Calvino MC, Llorca J, Garcia-Porrua C, et al. Henoch-Schonlein purpura in children from northwestern Spain: A 20-year epidemiologic and clinical study. Medicine (Baltimore). 2001;80(5):279-290.

13. Masuda M, Nakanishi K, Yoshizawa N, lijima K, Yoshikawa N. Group A streptococcal antigen in the glomeruli of children with Henoch-Schonelein nephritis. Am J Kidney Dis. 2003;41(2):366-370.

14. Yang $\mathrm{YH}$, Wang SJ, Chuang YH, Lin YT, Chiang BL. The level of IgA antibodies to human umbilical vein endothelial cells can be enhanced by TNF-a treatment in children with Henoch-Schönlein purpura. Clin Exp Immunol. 2002;130(2):352-357.

15. Grenda R. Nefropatia w przebiegu plamicy Henocha-Schönleina. Nefrol Dial Pol. 2008;12 (3):186-192.

16. Davin JC, Ten Berge IJ, Weening JJ. What the difference between IgA nephropathy and Henoch-Schönlein purpura nephritis? Kidney Int. 2001;59(3):823-834.

17. Gonzáles-Gay M, López-Mejías R, Pina T, Blanca R, Castaňeda S. IgA vasculitis: Genetics and clinical and therapeutic management. Curr Rheumatol Rep. 2018;20(5):24.

18. López-Mejías R, Genre F, Pérez BS, et al. HLA-DRB1 association with Henoch-Schonlein purpura. Arthritis Rheumatol. 2015;67(3):823-827.

19. Brogan PA. What's new in the aetiopathogenesis of vasculitis? Pediatr Nephrol. 2007;22(8):1083-1094.

20. Amoli MM, Thomson W, Hajeer AH, et al. HLA-B35 association with nephritis in Henoch-Schönlein purpura. J Rheumatol. 2002;29(5): 948-949.

21. Brogan P, Eleftheriou D, Dillon M.Small vessel vasculitis. Pediatr Nephrol. 2010;25(6):1025-1035.

22. Heineke $M H$, Ballering AV, Jamin A, Ben Mkaddem S, Monteiro RC, Van Egmond M. New insights in the pathogenesis of immunoglobulin A vasculitis (Henoch-Schönlein purpura). Autoimmunity Rev. 2017; 16(12):1246-1253.

23. Lau K, Suzuki H, Novak J, Wyatt RJ. Pathogenesis of Henoch-Schönlein purpura nephritis. Pediatr Nephrol. 2010;25(1):19-20.

24. Gharavi AG, Moldoveanu Z, Wyatt RJ, et al. Aberrant IgA1 glycosylation is inherited in familial and sporadic IgA nephropathy. J Am SoC Nephrol. 2008;19(5):1008-1014. 
25. Kiryluk K, Moldoveanu Z, Sanders JT, et al. Aberrant IgA1 glycosylation is inherited in pediatric IgA Nephropathy and in Henoch-Schönlein Purpura nephritis. Kidney Int.2011;80(1):79-87.

26. Suzuki H, Raska M, Yamada K, et al. Cytokines alter IgA1 O-glycosylation by disregulating C1GalT1 and ST6GalNAc-II enzymes. J Biol Chem. 2014;289(8):5330-5339.

27. Mizerska-Wasiak M, Gajewski Ł, Cichoń-Kawa K, et al. Serum GDIgA1 levels in children with IgA nephropathy and Henoch-Schönlein nephritis. Cent Eur J Immunol. 2018;43(2):162-167.

28. Moura IC, Acros-Fajardo M, Sadaka C, et al. Glycosylation and size of $\lg \mathrm{A} 1$ are essential for interaction with mesangial transferring receptor in IgA nephropathy. J Am Soc Nephrol. 2004;15(3):622-634.

29. Haddad E, Moura IC, Arcos-Fajardo M, et al. Enhanced expression of the CD71 mesangial IgA1 receptor in Berger disease and Henoch-Schönlein nephritis: Association between CD71 expression and IgA deposits. J Am Soc Nephrol. 2003;14(2):327-337.

30. Lai KN, Leung JC, Chan Ly, et al. Activation of podocytes by mesangial-derived TNF-alpha: glomerulo-podocytic communication in lgA nephropathy. Am J Physiol Renal Physiol. 2008;294(4):F945-F955.

31. Yang YH, Chuang YH, Wang LC, Huang HY, Gershwin ME, Chiang BL. The immunobiology of Henoch-Schönlein purpura. Autoimmun Rev. 2008;7(3):179-184.

32. Roos A, Bouwman LH, Van Gijlswijk-Janssen DJ, Faber-Krol MC, Stahl GL, Daha MR. Human IgA activates the complement system via the mannan-binding lectin pathway. J Immunol. 2001;167(5):2861-2868.

33. Narchi $H$. Risk of long term renal impairment and duration of follow up recommended for Henoch-Schönlein purpura with normal or minimal urinary findings: A systematic review. Arch Dis Child. 2005; 90(9):916-920.

34. Chan H, Tang YL, Lv XH, et al. Risk Factors Associated with renal involvement in childhood Henoch-Schönlein purpura: A meta-analysis. PLoS One. 2016;11(11):e0167346.

35. Halling SF, Söderberg MP, Berg UB. Henoch Schonlein nephritis: Clinical findings related to renal function and morphology. Pediatr Nephrol. 2005;20(1):46-51.

36. Ronkainen J, Nuutinen M, Koskimies O. The adult kidney 24 years after childhood Henoch-Schönlein purpura: A retrospective cohort study. Lancet. 2002;360(9334):666-670.

37. Hara M, Yanagihara T, Kihara I. Cumulative excretion of urinary podocytes reflects disease progression in IgA nephropathy and SchönleinHenoch purpura nephritis. Clin J Am Soc Nephrol. 2007;2(2):231-238.

38. Jelusic M, Sestan M, Cimaz R, Ozen S. Different histological classifications for Henoch-Schönlein purpura nephritis: Which one should be used? Pediatr Rheumatol Online J. 2019;17(1):10.

39. Nicoara O, Twombley K. Immunoglobulin A nephropthy and immu noglobulin A vasculitis. Pediatr Clin North Am. 2019;66(1):101-110.

40. Huang X, Wu J, Wu XW, et al. Significance of histological crescent for mation in patients with $\lg A$ vasculitis (Henoch-Schönlein purpura)related nephritis: $A$ cohort in the adult Chinese population. BMC Nephrol. 2018;19(1):334.

41. Davin JC. Henoch-Schönlein purpura nephritis: Pathophysiology, treatment, and future strategy. Clin J Am Soc Nephrol. 2011;6(3):679689.
42. Xu K, Zhang L, Ding J, et al. Value of the Oxford classification of IgA nephropathy in children with Henoch-Schönlein purpura nephritis. J Nephrol. 2018;31(2):279-286.

43. Huang $X, M a L$, Ren $P$, et al. Updated Oxford classification and the international study of kidney disease in children classification: Application in predicting outcome of Henoch-Schönlein purpura nephritis. Diagnostic Pathol. 2019; doi:10.1186/s13000-019-0818-0.

44. Koskela M, Ylinen E, Ukonmaanaho EM, et al. The ISKDC classification and a new semiquantitative classification for predicting outcomes of Henoch-Schönlein purpura nephritis. Pediatr Nephrol. 2017;32(7): 1201-1209.

45. Zaffanello M, Fanos V. Treatment-based literature of Henoch-Schönlein purpura nephritis in childhood. Pediatr Nephrol. 2009;24(10): 1901-1911.

46. Kidney Disease: Improving global outcomes (KDIGO). Henoch-Schönlein purpura. Kidney Int Suppl. 2012(11):218-220.

47. Hahn D, Hodson EM, Willis NS, Craig JC. Interventions for preventing and treating kidney disease in Henoch-Schönlein purpura (HSP). Cochrane Database Syst Rev. 2015;7(8):CD005128.

48. Dudley J, Smith G, Llewelyn-Edwards A, Bayliss K, Pike K, Tizard J. Randomised, double-blind, placebo-controlled trial to determine whether steroids reduce the incidence and severity of nephropathy in Henoch-Schonlein purpura (HSP). Arch Dis Child. 2013;98(10): 756-763.

49. Davin JC, Coppo R. Pitfalls in recommending evidence-based guidelines for protean disease like Henoch-Schonlein purpura nephritis. Pediatr Nephrol. 2013;28(10):1897-1903.

50. Salewski D, Ambruzs J, Appel G, et el. Clinical characteristics and treatment patterns of children and adults with IgA nephropathy or IgA vasculitis: Findings from CureGN Study. Kidney Int Rep.2018;3(6):1373-1384.

51. Koskela $M$, Jahnukainen $T$, Endén $K$, et al. Methylprednisolone or cyclosporine $a$ in the treatment of Henoch-Schönlein nephritis: A nationwide study. Pediatr Nephrol. 2019;34(8):1447-1456.

52. Ozen S, Marks SD, Brogan P, et al. European consensus-based recommendations for diagnosis and treatment of immunoglobulin A vasculitis - the SHARE initiative. Rheumatology (Oxford). 2019. doi:10. 1093/rheumatology/kez041

53. Goldstein AR, White RH, Akuse R, Chantler C. Long-term follow-up of childhood Henoch-Schonlein nephritis. Lancet. 1992;339(8788): 280-282.

54. Nussinovitch N, Elishkevitz K, Volovitz B, Nussinovitch M. Hypertension as a late sequela of Henoch-Schönlein purpura. Clin Pediatr. 2005;44(6):543-547.

55. Audemard-Verger A, Pillebout E, Guillevin L, Thervet E, Terrier B. IgA vasculitis (Henoch-Shönlein purpura) in adults: Diagnostic and therapeutic aspects. Autoimmun Rev. 2015;14(7):579-585.

56. Heleniak Z, Dębska-Ślizień A, Ptasińska-Perkowska A, Rutkowski B. Zapalenie naczyń związane z lgA (plamica Henocha-Shönleina) a nerki. Forum Nefro. 2014;7(3):159-168.

57. Kanaan N, Mourad G, Thervet E, et al. Recurrence and graft loss after kidney transplantation for Henoch-Schönlein purpura nephritis: A multicenter analysis. Clin J Am Soc Nephrol. 2011;6(7):1768-1772. 